\title{
Time-Domain Astrophysics with SuperWASP
}

\author{
A. J. Norton ${ }^{1}$ and the SuperWASP Consortium ${ }^{2}$ \\ ${ }^{1}$ Department of Physical Sciences, The Open University, Walton Hall, Milton Keynes, U.K. \\ email: a.j.norton@open.ac.uk \\ ${ }^{2}$ The SuperWASP project is funded and operated by Queen's University Belfast, the \\ Universities of Keele, St. Andrews and Leicester, the Open University, the Isaac Newton \\ Group, the Instituto de Astrofisica de Canarias, the South African Astronomical Observatory \\ and by STFC
}

\begin{abstract}
SuperWASP is the world's leading ground-based survey for transiting exoplanets. Its database now contains over 300 billion data points covering 30 million unique objects from 10 million images obtained over 1700 nights since 2004. In addition to having discovered 70 transiting exoplanets, SuperWASP enables long-baseline, high-cadence studies of variable stars to be performed. We summarise some of the studies already carried out, and look ahead to the prospects for studying periodic variables with varying periods. The science which is thus supported will include studies of the Blazhko effect in RR Lyræ stars, migrating starspots in rotational variables, third bodies in eclipsing binaries, and coalescing binary stars.
\end{abstract}

Keywords. Surveys, Catalogues, Stars: Binaries: Eclipsing, Stars: Rotation, Stars: Variables

\section{Introduction}

The Wide Angle Search for Planets (WASP) is the world's leading ground-based survey for transiting exoplanets (Pollacco et al. 2006), having discovered 70 to date (Enoch et al. 2011). It is comprised of two installations, on La Palma (Canary Islands) and at Sutherland (South Africa), and consists of 8 cameras per instrument on a single robotic mount. Each camera has a Canon lens of $111 \mathrm{~mm}$ aperture and $200 \mathrm{~mm}$ focal length, that is backed by a $2048 \times 2048$-pixel Andor CCD. The combination provides a $7^{\circ} .8 \times$ $7^{\circ} .8$ field of view and an image scale of $13^{\prime \prime} .7$ per pixel. Light curves are generated for all stars within the field within a magnitude range of $8<m_{v}<15$.

The SuperWASP database, whose public archive may be found at the web address http://wasp.le.ac.uk/public, holds over 300 billion data points covering 30 million unique objects, from 10 million images obtained over 1700 nights since 2004. The coverage of the survey is now virtually the entire sky, with the exception of the Galactic plane where the stellar density is too high to permit useful aperture-photometry of objects on account of the instrument's large pixel size.

In order to investigate stellar variability, positions of SuperWASP objects are first matched with the USNO-B1 catalogue. That enables light curves to be constructed for individual objects across the entire observing period. The stellar light curves so produced are sensitive to variability on time-scales from minutes to years, with a cadence as short as 30 seconds. Period searching on SuperWASP light curves uses a combination of a CLEANed power spectrum and a phase dispersion minimisation technique to identify periods in common between the two. Many systematic noise periods at $1 / n$ fractions of a sidereal day are apparent, and are caused by events such as temperature-dependent focus shifts which vary across the image plane. In addition, several sets of multiple objects 
display identical periods because of the large pixel size and issues with blending. Such effects complicate things, but nonetheless over 1 million light curves which are found display genuine periodic variability. Automated Neural Network classification of folded light curves based on their shape is on-going.

\section{Periodic Variable Stars Coincident with ROSAT Sources}

In a first attempt to investigate the variability of a manageable but interesting subset of the SuperWASP objects, the positions of the objects were cross-correlated against ROSAT X-ray all sky survey catalogues (1RXS \& 2RXP catalogues). That initial study (Norton et al. 2007) found 428 unique objects displaying periodic variability in their SuperWASP light curves and which were also coincident with ROSAT X-ray sources. We determined that there is a roughly $5 \%$ chance alignment between ROSAT sources and SuperWASP objects, and that at least $80 \%$ of those matches are likely to be real.

68 of the 428 objects just mentioned were previously-identified variable stars, 66 having known periods. They included 47 objects in the General Catalog of Variable Stars (GCVS), 17 objects discovered by the Robotic Optical Transient Search Experiment (ROTSE), and 2 discovered by the Semi-Automatic Variability Search (SAVS). They were classified as follows: 13 pre-main-sequence stars, $10 \mathrm{EA}$ (Algol type) binaries, $5 \mathrm{~EB}$ (Beta Lyræ type) binaries, $10 \mathrm{EW} \mathrm{(W} \mathrm{Ursa} \mathrm{Majoris} \mathrm{type)} \mathrm{binaries,} 6$ BY Dra systems, 5 RS CVn systems, 2 RR Lyræ stars, 15 Cepheid variables, 3 cataclysmic variables, 1 SuperSoft X-ray Source and 1 low-mass X-ray binary. (The Cepheid classifications given by ROTSE were probably mistaken).

Amongst the 360 newly-identified periodic variables, we demonstrated that most were likely to be rapid rotators of some sort (e.g., pre-main sequence stars, RS CVn stars, BY Dra stars), but the sample included many eclipsing binaries too. The project thus demonstrated the effectiveness of the SuperWASP survey for detecting photometric modulation over a range of time-scales amongst these moderately bright stars.

\section{Short-Period Eclipsing-Binary Candidates}

A second exploratory project was an investigation of some of the shortest-period variable objects revealed by the SuperWASP survey. Low-mass dwarf stars are very common, but their evolution in close binaries is poorly understood. In fact, a short period cut-off for eclipsing binaries with main-sequence components exists around a period of $\sim 0.22$ days, but few are known close to that limit. The cause of that cut-off is unclear; it is possibly linked to the finite age of the population.

To investigate this problem further, Norton et al. (2011) identified 5,600 periodic signals in the SuperWASP database in the period range 125-167 minutes, plus a further 17,300 signals close to $1 / 9 \mathrm{~d}$ ( $\sim 160$ minutes), 14,500 signals close to $1 / 10 \mathrm{~d}$ (144 minutes), and 9,300 signals close to $1 / 11 \mathrm{~d}$ ( $\sim 131$ minutes $)$ - most of which were false for the reasons outlined earlier. Contact binaries will generally be detected by our search technique at half their true orbital period, so the range searched corresponded to orbital periods between 250 minutes and 333 minutes. Visual examination of the light curves revealed 53 candidate eclipsing binaries (EW type) with broad maxima and narrow minima, only 5 of which were previously known. The rest of the objects display sinusoidal modulation (because they are rotational variables) or narrow maxima and broad minima (characteristic of pulsating variables). The process also allowed us to identify the shortest-period binary known that has dM components: GSC2314-0530 = 1SWASP J022050.85+332047.6, with a period of 277.4 minutes (or 0.1926 days). 
The orbital-period distribution produced by that sample increased the number of known short-period binaries (i.e., with orbital periods less than 0.23 days) by a factor of 6. Follow-up spectroscopic observations of these relatively bright candidates will allow the theory of the evolution of low-mass binary stars and their proposed mass-radius-period relationships to be tested further.

\section{Periodic Variables with Varying Periods}

Future research on the variable-star population revealed by SuperWASP will focus on those variable stars for which evidence of a varying period may be detected. That work is forming the basis of an STFC-funded $\mathrm{PhD}$ project. Four types of variability will be considered and sought:

\subsection{Varying period and/or amplitude of pulsating (RR Lyræ) stars}

The cause of this phenomenon in RR Lyræ stars, known as the Blazhko effect, is presently unknown. The long time-base and continuous coverage of the $>10,000$ RR Lyræ stars which SuperWASP will observe will enable the phenomenon to be investigated in unprecedented detail, and should offer new insights into its origin.

\subsection{Varying periods in stars displaying rotational modulation}

The surfaces of cool stars are often dominated by star spots, whose presence imparts a photometric variability to the stellar light curve as the star rotates. Star spots are expected to migrate in latitude with time. Many stars rotate differentially, giving rise to a variation in the observed rotation period and hence allowing investigation of the outer structure of the star. SuperWASP detects hundreds of thousands of stars displaying rotational modulation, and offers the prospect of statistical analysis of this behaviour across a variety of stellar spectral types.

\subsection{Third bodies in eclipsing binaries}

The $>10,000$ eclipsing binaries detected by SuperWASP offer an accurate "clock" by which to measure the orbital periods of binary motion. Some of these binary stars will be part of hierarchical triple systems and some may host circumbinary exoplanets. By searching for eclipsing binary periods which themselves vary cyclically with longer periods, the presence of third objects may be identified and characterized.

\subsection{Coalescing binaries}

Close eclipsing binary stars will lose angular momentum via a combination of magnetic braking and gravitational radiation. The very closest binaries will eventually merge, resulting in a single stellar core. Such a merger has been observed only once (Tylenda et al. 2011), in the star V1309 Sco. The long time-base offered by SuperWASP will allow us to search for other eclipsing binaries whose periods are systematically decreasing, and which may therefore be in or near the final stages of merger.

\section{References}

Enoch, B., et al. 2011, AJ, 142, 86

Norton, A. J., et al. 2007, Aछ A, 467, 785

Norton, A. J., et al. 2011, A\&A, 528, A90

Pollacco, D. L., et al. 2006, PASP, 118, 1407

Tylenda, R., et al. 2011, A\&SA, 528, A114 